\title{
Fraunhofer diffraction description in the approximation of the light field theory
}

\author{
Vladimir P. Budak ${ }^{\mathrm{a}, *}$, Dmitry S. Efremenko ${ }^{\mathrm{b}}$, Pavel A. Smirnov ${ }^{\mathrm{a}}$ \\ ${ }^{a}$ Department of Lighting Engineering, NRU MPEI, Moscow, Russia \\ ${ }^{b}$ Remote Sensing Technology Institute (IMF), German Aerospace Center (DLR), 82234 Oberpfaffenhofen, Germany
}

\begin{abstract}
The wavelength is that natural scale that determines the applicability domains of the ray approximation and the wave approximation of light. If the change of the radiation power spatial density is significant at the wavelength scale, we deal with the light diffraction phenomenon, which is a subject to the wave optics. Let us consider the diffraction phenomenon at the diaphragm. It is possible to distinguish the near zone with significant wave inhomogeneities (i.e. the Fresnel zone) and the far Fraunhofer diffraction zone, in which the wave becomes close to homogeneous (the so-called quasi-homogeneous) and the ray approximation is possible. The problem is that there is no explicit relationship between the radiance of the rays before and after diaphragm. Method for determining the boundary conditions for the radiance in the Fraunhofer zone through the radiance of the incident radiation is proposed in the paper. This approach for computing the radiance field in the Fraunhofer zone can be generalized to other problems of optics, thereby providing the possibility of using computationally efficient ray-approximation-based methods to determine the light fields.
\end{abstract}

Keywords: diffraction, geometrical optics, wave optics, photometry, quasi-heterogeneity

\section{Introduction}

Diffraction is considered as one of the main manifestations of the wave nature of light [1]. However, nowadays, this is most likely related to the school physics course, where it is common to antagonistically set the wave representation of light against the ray approximation, which is a consequence of the methodology of classical science in physics. We study any physical phenomenon or object by using a specific probe, tester, or test receiver. Still, in classical physics, it has always been assumed that the latter can always be made negligible, and thus, the properties of the object itself can be formulated. Using the quantum mechanics concepts, it became obvious that we cannot go beyond the description of the tester-object interaction, and in any physical phenomenon, there is some probe scale (related to the critical size), such that any attempt to reduce further the receiver's size violates the established physical description of the object.

A feature of optics is that two types of receivers can be distinguished, namely, quadratic and linear receivers. A quadratic receiver responds to the energy absorbed by it, while a linear one is a sort of antenna, the reaction of which is proportional to the field strength. Accordingly, each of the measurement processes generates its field: the quadratic receiver generates the light or radiation field, while the linear receiver generates the wavefield [2]. Essentially, the light field is the rays propagating in various directions along which power flows with spatial density $L(\mathbf{r}, \hat{\mathbf{l}}, t)$, i.e. the radiance of the light field at point $\mathbf{r}$, time $t$, in the direction $\hat{\mathbf{l}}$. The wave field characterizes the spatial-temporal distribution of the electric field intensity $\mathbf{E}(\mathbf{r}, t)$. Its structure can be represented as a superposition of random waves in space.

As the energy is a quadratic quantity concerning the electric field, then all the properties of the light field can be expressed in terms of the properties of the wave field. However, in the optical spectral range, only

\footnotetext{
${ }^{*}$ Corresponding author

Email address: budakvp@gmail.com (Vladimir P. Budak)
} 


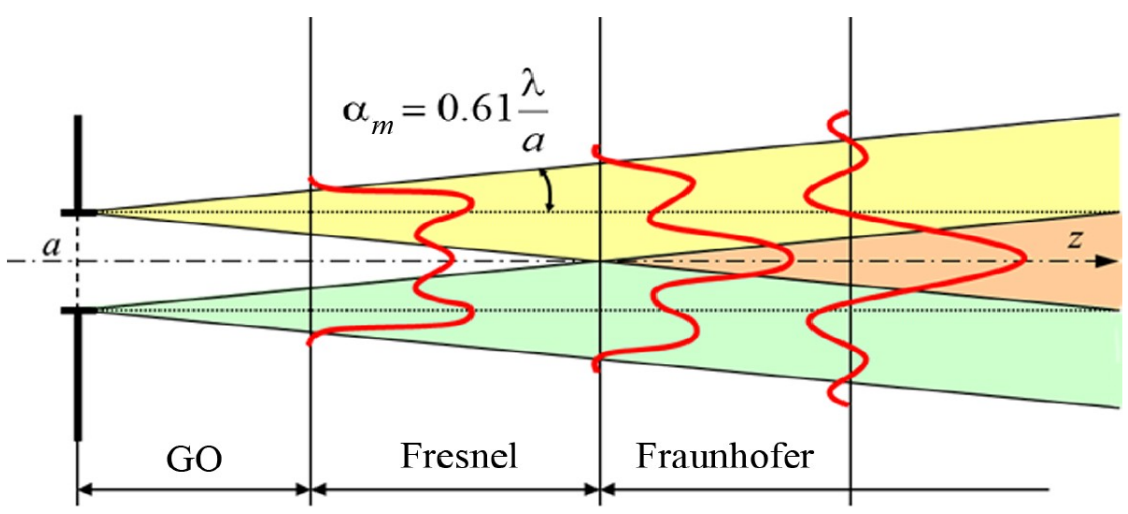

Fig. 1. Distribution of the irradiance (red line) as a function of the distance to the diaphragm aperture $z$

quadratic receivers exist. A linear receiver can be set up by placing an optical device, such as an aperture, in front of the quadratic receiver. If the diaphragm dimensions are smaller than a specific size parameter $\lambda$, then the field after the diaphragm will be a wave one, however, we can still measure its characteristics only by using a quadratic receiver. In the simplest case of a homogeneous monochromatic wave $\lambda$ is the wavelength.

If the amplitude (i.e. intensity) of the wavefield and the energy density (i.e. power) of the light field does not change in space, then the field is called homogeneous, and both description models are essentially equivalent to each other: at each point in space, the beam is perpendicular to the wavefront. The wavefront acts like a function, which describes all possible ray paths. If the field is inhomogeneous, especially in the case when the spatial scale of the field change becomes of the order or less than 1 , then the ray approximation is not valid anymore. In the narrow sense of the word, this phenomenon is commonly called diffraction.

The field of the diffracted wave right after the diaphragm can be represented as a superposition [1] of a strongly inhomogeneous component, decreasing with distance $r$ from the diaphragm faster than $1 / r$, and a quasihomogeneous component, slowly changing over the scale of $\lambda$ and decreasing as $1 / r$. Therefore, the field at the diaphragm is strongly inhomogeneous (that is the Fresnel zone), but at a certain distance, the Fraunhofer zone can be distinguished, where the wave is again quasihomogeneous and can be treated in the framework of the ray approximation. In a general case, the field pattern after diffraction at a circular aperture of radius $a$ can be represented, as shown in Fig. 1.

The analysis is based on the idea that diffraction generates at each point of the diaphragm a divergent beam with a divergence angle $\alpha_{m} \sim \lambda / a$, corresponding to the size of the Airy disk [1]. If the overlapping of spots of beams at the distance $z$ from the diaphragm aperture is $z \alpha_{m} \approx z \lambda / a \ll a$, then the impact of diffraction is negligible, and the field can be described in term of the ray approximation, wherein the radiance of the rays passing through the diaphragm is equal to the radiance of the incident radiation. If the overlapping of the spots is significant (i.e. $z \lambda / a \approx a$ ), which corresponds to the Fresnel zone, the field is determined by the interference of the beams generated at each point of the diaphragm. It becomes substantially inhomogeneous and the ray approximation cannot be applied. In the Fraunhofer zone we have $z \lambda / a \gg a$ and the beams generated at all diaphragm points are strongly mixed, the field becomes quasi-homogeneous, and the ray approximation comes into play. However, the radiance of the rays is no longer determined by the radiance of the incident, but rather by the laws of diffraction.

The problem is that the connection between the rays before and after the diaphragm is not apparent. It can be determined only through the properties of the wavefield right after the diaphragm [3]. The goal of this paper is to derive a relationship of the spatial-angular radiance distributions $L(\mathbf{r}, \hat{\mathbf{l}})$ of stationary light fields before and after the diaphragm. 
Fig. 2. The exit pupil of the optical system

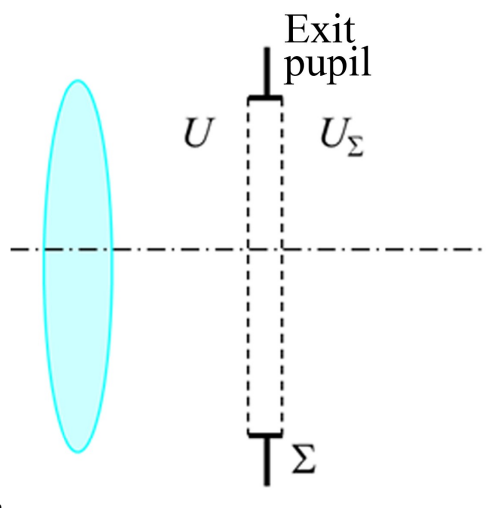

\section{Wave diffraction at the exit pupil of the optical system}

According to the Abbe principle (Abbe Ernst, 1840-1905) [4], the structure of the optical image can be studied in the geometric optics approximation considering the wave diffraction at the exit pupil. Let us consider a sharp image from a point lying on the optical axis. As we must have a spherical wave focusing to the image point in the plane of analysis, the wave in the plane of analysis is quasi-homogeneous.

Let the complex amplitude of the wave passing though the optical system (OS) in the plane of the exit pupil be $U(\mathbf{r})$, where $\mathbf{r}$ is the radius-vector in the plane of exit pupil $\Sigma$ (as shown in Fig. 2). Then the field immediately after the pupil is expressed through the pupil function $\Theta(\mathbf{r})$ :

$$
U_{\Sigma}(\mathbf{r})=\Theta(\mathbf{r}) U(\mathbf{r})
$$

where the pupil function is $\Theta(\mathbf{r})= \begin{cases}1, & \mathbf{r} \in \Sigma, \\ 0, & \mathbf{r} \notin \Sigma .\end{cases}$

In the view of Eq. (1), the expression for the correlation function of the field right after the exit pupil reads:

$$
\begin{aligned}
& \Gamma_{\Sigma}\left(\mathbf{r}_{1}, \mathbf{r}_{2}\right)=\left\langle U_{\Sigma}\left(\mathbf{r}_{1}\right) U_{\Sigma}^{*}\left(\mathbf{r}_{2}\right)\right\rangle= \\
& \Theta\left(\mathbf{r}_{1}\right) \Theta\left(\mathbf{r}_{2}\right)\left\langle U\left(\mathbf{r}_{1}\right) U^{*}\left(\mathbf{r}_{2}\right)\right\rangle=\Theta\left(\mathbf{r}_{1}\right) \Theta\left(\mathbf{r}_{2}\right) \Gamma_{o}\left(\mathbf{r}_{1}, \mathbf{r}_{2}\right)
\end{aligned}
$$

where $\Gamma_{o}\left(\mathbf{r}_{1}, \mathbf{r}_{2}\right)$ is the correlation function of radiation passing though the OS on the plane of exit pupil.

Introducing the local coordinates in the plane of the exit pupil $\Sigma$, i.e.

$$
\mathbf{R}=\frac{\mathbf{r}_{1}+\mathbf{r}_{2}}{2}, \quad \rho=\mathbf{r}_{1}-\mathbf{r}_{2}, \quad \mathbf{r}_{1}=\mathbf{R}+\frac{\rho}{2}, \quad \mathbf{r}_{2}=\mathbf{R}-\frac{\rho}{2}
$$

yields

$$
\Gamma_{\Sigma}(\mathbf{R}, \rho)=\Gamma_{o}(\mathbf{R}, \rho) \Theta\left(\mathbf{R}+\frac{\rho}{2}\right) \Theta\left(\mathbf{R}-\frac{\rho}{2}\right) .
$$

Using our assumption that the field before the exit pupil can be computed in the ray approximation (which is equivalent to the quasi-homogeneous field assumption), we can express $\Gamma_{o}(\mathbf{R}, \rho)$ though the generalized radiance in the Wigner spectrum form $[3,5]$ :

$$
\Gamma_{o}(\mathbf{R}, \rho)=\oint L_{o}(\mathbf{R}, \hat{\mathbf{l}}) e^{-i k \hat{\mathbf{l}} \rho} d \hat{\mathbf{l}}
$$

where $L_{o}(\mathbf{R}, \hat{\mathbf{l}})$ is the radiance of the radiation passing through the OS in the plane of the exit pupil.

Then the radiance of the quasi-homogeneous part after the exit pupil, which forms the image in the plane 


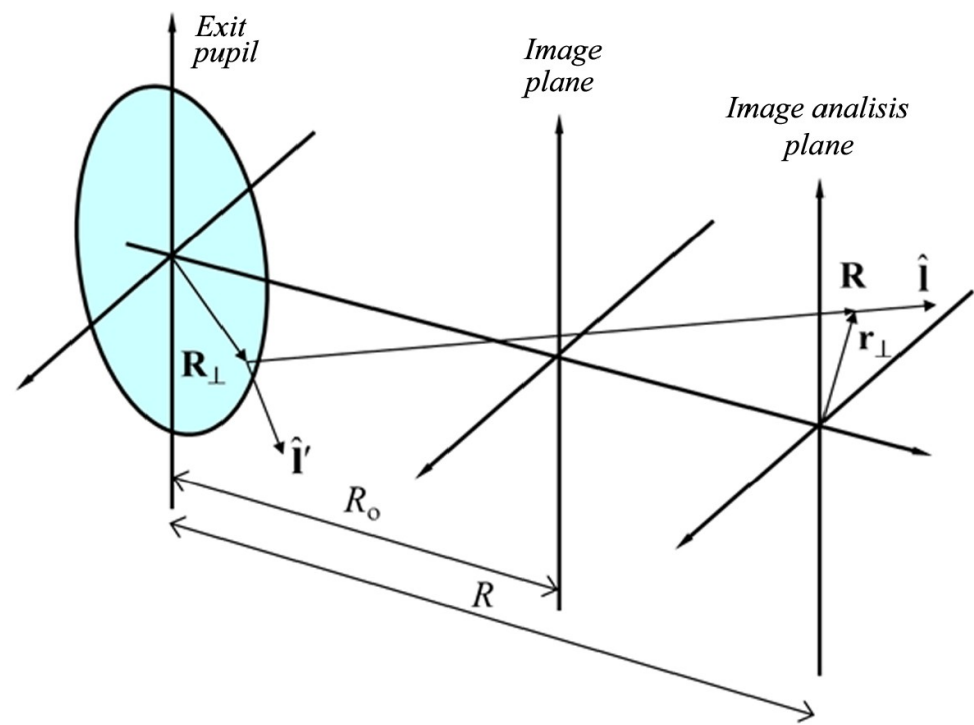

Fig. 3. The scheme of image formation in the optical system, bounded by diffraction

of analysis, reads as follows [3]:

$$
L\left(\mathbf{R}, \mathbf{l}_{\perp}^{\prime}\right)=\left(\frac{k}{2 \pi}\right)^{2} l_{z} \int \Gamma_{\Sigma}(\mathbf{R}, \rho) e^{i k \mathbf{l}_{\perp}^{\prime} \rho} d^{2} \rho,
$$

where $\mathbf{l}_{\perp}^{\prime}$ is the vector of projection $\mathbf{l}$ onto the normal to the image plane, while $\hat{\mathbf{l}}$ is defined according to Fig. 3 as follows:

$$
\hat{\mathbf{l}}=\frac{\mathbf{r}_{\perp}-\left(\mathbf{R}_{\perp}+R \hat{\mathbf{z}}\right)}{\sqrt{\left(\mathbf{r}_{\perp}-\mathbf{R}_{\perp}\right)^{2}+R^{2}}} .
$$

Substituting Eq. (4) in Eq. (6) gives

$$
\begin{aligned}
L\left(\mathbf{R}, \mathbf{l}_{\perp}^{\prime}\right)= & \left(\frac{k}{2 \pi}\right)^{2} l_{z} \oint L_{o}(\mathbf{R}, \hat{\mathbf{l}}) \int \Theta\left(\mathbf{R}+\frac{\rho}{2}\right) \Theta\left(\mathbf{R}-\frac{\rho}{2}\right) \\
& \times \exp \left(-i k\left(\mathbf{l}_{\perp}^{\prime}-\mathbf{l}_{\perp}\right) \rho_{\perp}\right) d^{2} \rho d \hat{\mathbf{l}} .
\end{aligned}
$$

Introducing

$$
\begin{aligned}
h\left(\mathbf{l}_{\perp}^{\prime}-\mathbf{l}_{\perp}\right)= & \left(\frac{k}{2 \pi}\right)^{2}\left(\frac{l_{z}}{l_{z}^{\prime}}\right) \int \Theta\left(\mathbf{R}+\frac{\rho}{\mathbf{2}}\right) \\
& \times \Theta\left(\mathbf{R}-\frac{\rho}{\mathbf{2}}\right) \exp \left(-i k \rho\left(\mathbf{l}_{\perp}^{\prime}-\mathbf{l}_{\perp}\right)\right) d^{2} \rho
\end{aligned}
$$

Eq. (8) can be written as a convolution:

$$
L\left(\mathbf{R}, \mathbf{l}_{\perp}^{\prime}\right)=\int L_{o}\left(\mathbf{R}, \mathbf{l}_{\perp}\right) h\left(\mathbf{l}_{\perp}^{\prime}-\mathbf{l}_{\perp}\right) d^{2} \mathbf{l}_{\perp} .
$$

Thus, the wave diffraction at the exit pupil acquires a clear ray interpretation of light scattering, while $h\left(\mathbf{l}_{\perp}^{\prime}-\mathbf{l}_{\perp}\right)$ is a function of light scattering. 


\section{Optical transfer function of an ideal optical system}

To validate the derived relations, let us consider the optical transfer function (OTF) of a non-aberrational thin lens, the frame of which also serves as the exit pupil in the presence of defocusing. The technique of computing the OTF of such a system is well known from wave optics [1]. For this, we consider an isotropic point source with a luminous intensity $I_{o}$ on the optical axis having a stigmatic image in the analysis plane at a distance $R_{o}$ along the optical axis from the plane of the exit pupil (as shown in Fig. 3). The irradiation distribution from it will be the point scattering function (PSF), the Fourier transform of which provides the desired OTF. The radiance of the beam in this case on the plane of the diaphragm can be expressed as follows:

$$
L_{o}\left(\mathbf{R}_{\perp}, \mathbf{l}_{\perp}^{\prime}\right)=I_{o} \delta\left(\mathbf{l}_{\perp}^{\prime}+\frac{\mathbf{R}_{\perp}}{R_{o}}\right) .
$$

Substituting Eq. (11) into Eq. (8) and expressing the result using coordinates $\mathbf{R}_{\perp}, \mathbf{l}_{\perp}$, we obtain

$$
\begin{aligned}
L_{\Sigma}\left(\mathbf{R}_{\perp}, \mathbf{l}_{\perp}\right)= & I_{o}\left(\frac{k}{2 \pi}\right)^{2}\left(\frac{l_{z}}{l_{z}^{\prime}}\right) \int \Theta\left(\mathbf{R}_{\perp}+\frac{\rho}{2}\right) \\
& \times \Theta\left(\mathbf{R}_{\perp}-\frac{\rho}{2}\right) \exp \left\{i k \rho\left(\mathbf{l}_{\perp}+\frac{\mathbf{R}_{\perp}}{R_{o}}\right)\right\} d^{2} \rho .
\end{aligned}
$$

Here we made use of the following geometrical properties (see Fig. 3):

$$
\begin{aligned}
& \mathbf{l}_{\perp} \simeq \frac{\mathbf{r}_{\perp}-\mathbf{R}_{\perp}}{R}, \quad \mathbf{l}_{\perp}+\frac{\mathbf{R}_{\perp}}{R_{o}}=\frac{\mathbf{r}_{\perp}}{R}+\beta \frac{\mathbf{R}_{\perp}}{R}, \\
& \beta=\frac{R-R_{o}}{R_{o}}, \quad d^{2} l_{\perp} \approx \frac{d^{2} R}{R^{2}} .
\end{aligned}
$$

The expression for the irradiance on the plane of analysis reads as

$$
E\left(\mathbf{r}_{\perp}\right)=\int_{\left(\Omega_{+}\right)} L_{\Sigma}\left(\mathbf{r}_{\perp}, \mathbf{l}_{\perp}\right) l_{z} d \hat{\mathbf{l}}=\int_{\left(\Omega_{+}\right)} L_{\Sigma}\left(\mathbf{r}_{\perp}, \mathbf{l}_{\perp}\right) l_{z} d^{2} l,
$$

or, making use of Eq. (12):

$$
\begin{aligned}
E\left(\mathbf{r}_{\perp}\right)= & \frac{I_{o}}{R^{2}}\left(\frac{k}{2 \pi}\right)^{2} \int \Theta\left(\mathbf{R}_{\perp}+\frac{\rho}{2}\right) \Theta\left(\mathbf{R}_{\perp}-\frac{\rho}{2}\right) \\
& \times \exp \left\{i k \rho\left(\frac{\mathbf{r}_{\perp}}{R}+\beta \frac{\mathbf{R}_{\perp}}{R}\right)\right\} d^{2} \rho d^{2} R_{\perp},
\end{aligned}
$$

Here it is taken into account that $l_{z} / l^{\prime} \approx 1$ under paraxial optics conditions.

OTF OS is the Fourier transform of irradiance distribution:

$$
H(\nu)=\int E\left(\mathbf{r}_{\perp}\right) e^{-i \nu \mathbf{r}_{\perp}} d^{2} r_{\perp},
$$

and using Eq. (15), we obtain

$$
\begin{aligned}
H(\nu)= & \frac{I_{o}}{R^{2}}\left(\frac{k}{2 \pi}\right)^{2} \int \Theta\left(\mathbf{R}_{\perp}+\frac{\rho}{2}\right) \Theta\left(\mathbf{R}_{\perp}-\frac{\rho}{2}\right) \\
& \times \exp \left\{i k \rho\left(\frac{\mathbf{r}_{\perp}}{R}+\beta \frac{\mathbf{R}_{\perp}}{R}\right)-i \nu \mathbf{r}_{\perp}\right\} d^{2} r_{\perp} d^{2} \rho d^{2} R_{\perp} .
\end{aligned}
$$




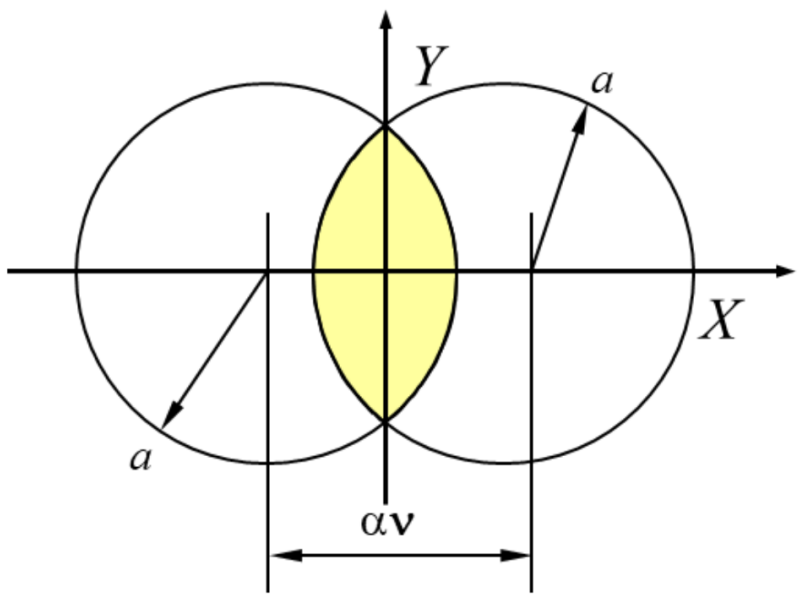

Fig. 4. To definition of the limits of integration in Eq. (22)

Note one obvious formula:

$$
\delta\left(\frac{k \rho}{R}-\nu\right)=\int \exp \left\{i \mathbf{r}_{\perp}\left(\frac{k \rho}{R}-\nu\right)\right\} d^{2} r_{\perp},
$$

which can transform Eq. (17) to the following form:

$$
H(\nu)=I_{o} \int \Theta\left(\mathbf{R}_{\perp}+\frac{\alpha \nu}{2}\right) \Theta\left(\mathbf{R}_{\perp}-\frac{\alpha \nu}{2}\right) \exp \left(i \beta \nu \mathbf{R}_{\perp}\right) d^{2} R_{\perp},
$$

where making use of Eq. (18)

$$
\alpha=\frac{R}{k}=\frac{\lambda R}{2 \pi} .
$$

Let us compute OTF of the optical system for zero spatial frequency:

$$
H(0)=I_{o} \int \Theta\left(R_{\perp}\right) d^{2} R_{\perp}=\pi a^{2} I_{o}
$$

Note that $H(0)$ corresponds to the transmittance of the optical system.

Then we can express relative OTF (i.e. the modulation transmission function) as follows:

$$
\begin{aligned}
T(\nu)= & \frac{H(\nu)}{H(0)}=\frac{1}{\pi a^{2}} \int \Theta\left(\mathbf{R}_{\perp}+\frac{\alpha \nu}{2}\right) \\
& \times \Theta\left(\mathbf{R}_{\perp}-\frac{\alpha \nu}{2}\right) \exp \left(i \beta \nu \mathbf{R}_{\perp}\right) d^{2} R_{\perp} .
\end{aligned}
$$

Let us consider the impact of defocusing on the OTF of the optical system. From Fig. 4, it follows that

$$
\left(x-\frac{\alpha \nu}{2}\right)^{2}+y^{2}=a^{2}
$$

and this allows to define the integration limits in Eq. (22):

$$
x_{1}=\frac{\alpha \nu}{2}-\sqrt{a^{2}-y^{2}}, \quad x_{2}=-\frac{\alpha \nu}{2}+\sqrt{a^{2}-y^{2}}, \quad y_{12}= \pm \sqrt{a^{2}-\frac{\alpha^{2} \nu^{2}}{4}},
$$


and express the integral as

$$
T(\nu)=\frac{2}{\pi a^{2}} \int_{0}^{\sqrt{a^{2}-\left(\frac{\alpha \nu}{2}\right)^{2}}} \quad \int_{\frac{\alpha \nu}{2}-\sqrt{a^{2}-y^{2}}}^{-\frac{\alpha \nu}{2}+\sqrt{a^{2}-y^{2}}} e^{i \beta \nu x} d x d y .
$$

Note that the integration over $d x$ can be easily computed:

$$
T(\nu)=\frac{2}{\pi a^{2}} \int_{0}^{\sqrt{a^{2}-\left(\frac{\alpha \nu}{2}\right)^{2}}} \sin \left[\beta \nu\left(\sqrt{a^{2}-y^{2}}-\frac{\alpha \nu}{2}\right)\right] d y .
$$

The integral takes a simpler form for further analysis,

$$
T(w)=\frac{4}{\pi \gamma w} \int_{0}^{\sqrt{1-\left(\frac{w}{2}\right)^{2}}} \sin \left[\gamma w\left(\sqrt{1-t^{2}}-\frac{w}{2}\right)\right] d t
$$

if we switch to the following dimensionless variables:

$$
\begin{aligned}
& w=\frac{\alpha \nu}{a}=\frac{R \nu}{a k}=\frac{R \nu}{\zeta}, \quad t=\frac{y}{a} \\
& \gamma=\frac{\beta a^{2}}{\alpha}=\frac{\beta k a^{2}}{R}=\beta \zeta \frac{a}{R}, \quad \zeta=k a=\frac{2 \pi a}{\lambda} .
\end{aligned}
$$

Fig. 5 shows the OTFs for several values of relative defocusing parameters $\gamma$.

Note that if there is no defocusing, which is equivalent to $\beta \rightarrow 0$ and, hence, $\gamma \rightarrow 0$, we have

$$
\lim _{\gamma \rightarrow 0} \frac{1}{\gamma w} \sin \left[\gamma w\left(\sqrt{1-t^{2}}-\frac{w}{2}\right)\right]=\sqrt{1-t^{2}}-\frac{w}{2}
$$

and Eq. (27) is reduced to the expression for the OTF for the OS with diffraction at the exit pupil:

$$
T(w)=\frac{4}{\pi} \int_{0}^{\sqrt{1-\left(\frac{w}{2}\right)^{2}}}\left(\sqrt{1-t^{2}}-\frac{w}{2}\right) d t=\frac{2}{\pi}\left[\arccos \frac{w}{2}-\frac{w}{2} \sqrt{1-\frac{w^{2}}{4}}\right],
$$

which completely corresponds to the formula derived in the framework of the diffraction theory [6-8].

In [7], Eq. (30) is expressed in terms of Bessel functions, which allows one to obtain the asymptotic formula for $T(w)$ for large values of $\gamma$. It is straightforward to see that the impact of diffraction blurring becomes negligible when the defocusing parameter increases. At the same time, the blur function for a point is defined by the geometrical optics, and the blur spot turns into a uniformly illuminated disk. In this case, the OTF as a Fourier transform from a uniformly luminous disk is given by a first-order Bessel function for its argument.

\section{Conclusion}

We have proposed a method for determining the relationship between the radiance of the light fields before the diaphragm and in the Fraunhofer zone after the diaphragm. This method can describe all phenomena related to image formation in an ideal optical system. Therefore, for analyzing the image in the optical system, the computationally efficient ray approximation can be applied, where the diaphragms are treated as scattering elements with the corresponding point spread functions (PSFs) according to Eq. (9). Moreover, since the Fraunhofer zone is of considerable practical interest, this approach can be generalized and developed for other problems. For example, nowadays, scattering by particles of complex shape is treated not according to Mie theory [9], but rather based on geometrical optics, in which the wave effects (e.g., diffraction, surface 


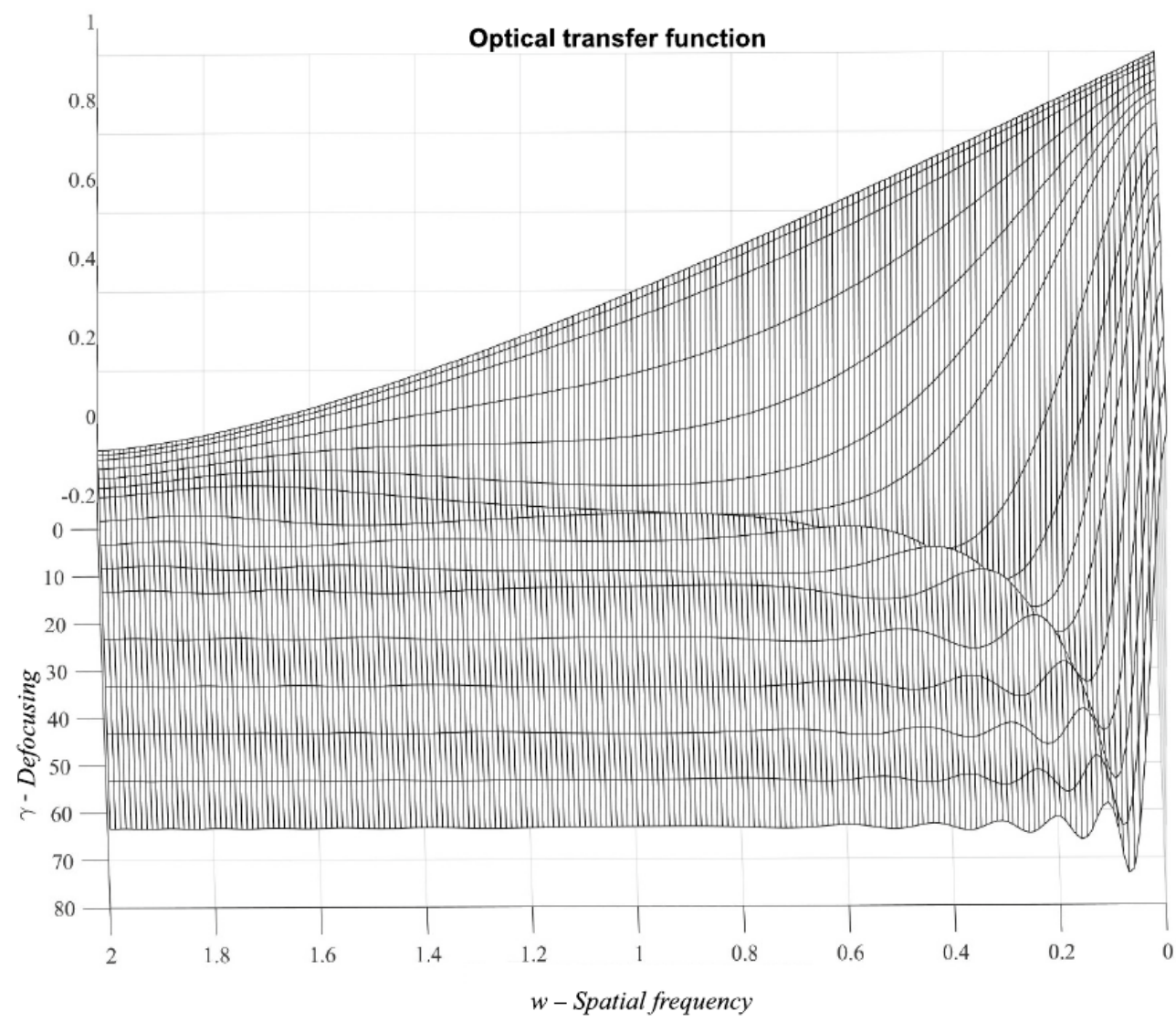

Fig. 5. Influence of defocusing on OTF of the ideal optical system, bounded by diffraction

wave, and edge effect) are neglected. By imposing new boundary conditions for the scattered rays in the Fraunhofer zone, it is possible to include these effects in the ray optics models.

\section{References}

1. M. Born, E. Wolf, Principles of Optics, Cambridge University Press, 1999. doi:10.1017/cbo9781139644181.

2. V. Budak, Theory of the light field / Section 2.1., in: Y. Eisenberg, G. Boos (Eds.), Reference Book on Lighting Engineering, Editorial Board of the journal "Lighting Engineering, 2020.

3. A. L.A., K. Yu.A., Radiation Transport Theory: Statistical and Wave Effects, Amsterdam: OPA, 1996.

4. E. Abbe, Beiträge zur theorie des mikroskops und der mikroskopischen wahrnehmung, Archiv für Mikroskopische Anatomie 9 (1) (1873) 413-468. doi:10.1007/bf02956173.

5. E. Wigner, On the quantum correction for thermodynamic equilibrium, Physical Review 40 (5) (1932) 749-759. doi: 10.1103/physrev.40.749.

6. H. Hopkins, On the diffraction theory of optical images, Proceedings of the Royal Society of London. Series A. Mathematical and Physical Sciences 217 (1130) (1953) 408-432. doi:10.1098/rspa.1953.0071.

7. H. Hopkins, The frequency response of a defocused optical system, Proceedings of the Royal Society of London. Series A. Mathematical and Physical Sciences 231 (1184) (1955) 91-103. doi:10.1098/rspa.1955.0158.

8. W. Steel, The defocused image of sinusoidal gratings, Optica Acta: International Journal of Optics 3 (2) (1956) 65-74. doi: $10.1080 / 713823633$.

9. G. Mie, Beiträge zur optik trüber medien, speziell kolloidaler metallösungen, Annalen der Physik 330 (3) (1908) $377-445$. doi:10.1002/andp.19083300302. 